\title{
A MULTIVARIATE ARITHMETIC FUNCTION OF COMBINATORIAL AND TOPOLOGICAL SIGNIFICANCE
}

\author{
VALERY A. LISKOVETS
}

\begin{abstract}
We investigate properties of a multivariate function $E\left(m_{1}, m_{2}, \ldots, m_{r}\right)$, called orbicyclic, that arises in enumerative combinatorics in counting non-isomorphic maps on orientable surfaces. $E\left(m_{1}, m_{2}, \ldots, m_{r}\right)$ proves to be multiplicative, and a simple formula for its calculation is provided. It is shown that the necessary and sufficient conditions for this function to vanish is equivalent to familiar Harvey's conditions that characterize possible branching data of finite cyclic automorphism groups of Riemann surfaces.
\end{abstract}

\section{Introduction}

Let $\left(m_{1}, m_{2}, \ldots, m_{r}\right)$ be a tuple of $r \geq 0$ positive integers and $m=$ $\operatorname{lcm}\left(m_{1}, m_{2}, \ldots, m_{r}\right)$, where $m:=1$ for $r=0$ (an empty tuple). Introduce the following multivariate function

$$
E=E\left(m_{1}, m_{2}, \ldots, m_{r}\right):=\frac{1}{M} \sum_{k=1}^{M} \Phi\left(k, m_{1}\right) \Phi\left(k, m_{2}\right) \cdots \Phi\left(k, m_{r}\right)
$$

$(E(\emptyset)=1)$, where $m \mid M, M>0$, and $\Phi(k, n)$ stands for the von Sterneck function:

$$
\Phi(k, n):=\frac{\phi(n)}{\phi\left(\frac{n}{(k, n)}\right)} \mu\left(\frac{n}{(k, n)}\right) .
$$

Here $(k, n)$ denotes the greatest common divisor of $k$ and $n$ and $\mu(n)$ and $\phi(n)$ are the Möbius and Euler functions respectively. According to O. Hölder (see, e.g., Apo76, Ch. 8], SSiv89, Ch.IX]), $\Phi(k, n)$ coincides with the Ramanujan trigonometric sum:

$$
\Phi(k, n)=C_{n}(k)
$$

where

$$
C_{n}(k):=\sum_{\substack{d(\bmod n) \\(d, n)=1}} \exp \left(\frac{2 i k d}{n}\right)
$$

with the summation over a reduced residue system modulo $n$. The function $C_{n}(k)$ (in the literature it has diverse designations such as $C(k, n)$ ) satisfies

Date: November 27, 2009.

1991 Mathematics Subject Classification. Primary: 11A25. Secondary: 05A15, 05C30, 11B75, 20H10, 30F20.

Key words and phrases. Ramanujan trigonometric sum, von Sterneck function, Jordan function, multiplicative function, map enumeration, unrooted map on orientable surface, cyclic group action, automorphism of Riemann surface, Harvey's conditions, orbifold, order-preserving epimorphism. 
the familiar Ramanujan's identity (loc. cite):

$$
C_{n}(k)=\sum_{d \mid(k, n)} d \mu\left(\frac{k}{d}\right) .
$$

Note that $E\left(m_{1}, m_{2}, \ldots, m_{r}\right)$ does not depend on $M$. Indeed, by (2), $\Phi\left(k, m_{j}\right)$ is a periodic function of $k$ modulo the second variable and, a fortiori, modulo $m$. Thus $\prod_{j} \Phi\left(k, m_{j}\right)$ as a function of $k$ is periodic modulo $m$ as well. So, in (1) we may put $M=m$. Now, $E$ is a symmetric function of its arguments, and we might speak about the (multi-)set of arguments instead of a tuple of them. Since $\Phi(k, 1)=1$ we may restrict $m_{j}$ to values greater 1 , that is,

$$
E\left(m_{1}, m_{2}, \ldots, m_{r-1}, 1\right)=E\left(m_{1}, m_{2}, \ldots, m_{r-1}\right) .
$$

This plays an important rôle in computational formulae for $E$ and applications. Tuples of arguments not containing 1 are called reduced.

$E\left(m_{1}, m_{2}, \ldots, m_{r}\right)$ is an essentially multivariate function in the sense that it is trivial for $r=0,1$. Moreover, for $r=2$ it vanishes for unequal arguments and coincides with the Euler function otherwise. Later we will see that $E\left(m_{1}, m_{2}, \ldots, m_{r}\right)$ is always non-negative and integer.

The function $E$ has been introduced by A. Mednykh and R. Nedela in 2004 (see [MeN06]) in the context of enumerative combinatorics: it plays a crucial rôle in counting maps on orientable surfaces up to orientationpreserving isomorphism, via the calculation of certain epimorphisms from the fundamental group of orbifolds onto cyclic groups (see Sect. 3). Therefore this "orbicyclic" arithmetic function (as we called it in [Lis05]) deserves a detailed investigation by its own right.

Here we study some basic properties of $E\left(m_{1}, m_{2}, \ldots, m_{r}\right)$. First of all we analyze the prime-power case and establish a simple sum-free formula for $E\left(p^{a_{1}}, p^{a_{2}}, \ldots, p^{a_{r}}\right), p$ prime. It is determined by three parameters (apart from $p$ ), denoted $r$ (reduced), $s$ and $v$, only one of which ( $s$, the multiplicity of the highest power) is responsible for its vanishing. In the general case we show that $E\left(m_{1}, m_{2}, \ldots, m_{r}\right)$ is a multiplicative function of all its arguments. Both results provide a simple explicit formula for its calculation. The most valuable property of $E\left(m_{1}, m_{2}, \ldots, m_{r}\right)$ established here is the necessary and sufficient conditions of its non-vanishing. We show that they are equivalent (due to the above-mentioned connection with the enumeration of epimorphisms) to the well-known conditions discovered by W. Harvey [Har66] (cf. also BuC99]) that specify the possible actions of finite cyclic automorphism groups of Riemann surfaces. Therefore the function $E\left(m_{1}, m_{2}, \ldots, m_{r}\right)$ may be considered as a fruitful enumerative refinement of Harvey's theorem, bringing a new insight into this theory.

The familiar Jordan arithmetic function also participates in the mentioned enumeration; we discuss briefly some other links between this function and enumerative combinatorial group theory.

For the reader's convenience, the paper contains rather numerous (although restricted) references to relevant publications in the three main topics we deal with: number theory (arithmetic functions), algebraic topology 
(automorphisms of Riemann surfaces), and algebraic and enumerative combinatorics (map theory and map enumeration). For a general material concerning these topics and the notions used in the present paper, the reader is referred to, resp., Apo76, BuEGG90 and CoM92.

\section{Function $E\left(m_{1}, m_{2}, \ldots, m_{r}\right)$ and its properties}

2.1. Primary case. $\Phi(k, n)$ is a multiplicative function of $n$ which is determined by the following well-known (and easily provable) formula; see, e.g., McC60]:

Lemma 2.1. For $p$ prime and $a \geq 1$,

$$
\Phi\left(k, p^{a}\right)=\left\{\begin{aligned}
(p-1) p^{a-1} & \text { if } p^{a} \mid k \\
-p^{a-1} & \text { if } p^{a} \nmid k, p^{a-1} \mid k \\
0 & \text { otherwise. }
\end{aligned}\right.
$$

This is an important result for calculating $E\left(m_{1}, m_{2}, \ldots, m_{r}\right)$ explicitly. As we will see below, the function $E\left(m_{1}, m_{2}, \ldots, m_{r}\right)$ is always non-negative, unlike $\Phi(k, n)$ (and it often vanishes like $\Phi(k, n)$ ).

At first, we consider the prime power case: $m=p^{a}$. Let $\left(m_{1}, m_{2}, \ldots, m_{r}\right)$ be a reduced tuple. Then $m_{j}=p^{a_{j}}, j=1,2, \ldots, r$, where without loss of generality we assume that

$$
a_{1}=a_{2}=\ldots=a_{s}=a>a_{s+1} \geq a_{s+2} \cdots \geq a_{r}>0,
$$

where $r \geq s \geq 1$. Denote

$$
v:=\sum_{j=2}^{r}\left(a_{j}-1\right)=\sum_{j=1}^{r} a_{j}-r-a+1, \quad v \geq 0 .
$$

The parameters $s(p)=s$ and $v(p)=v$, where $p \mid m$, can be defined for an arbitrary tuple of variables $\left(m_{1}, m_{2}, \ldots, m_{r}\right)$ as well (see Sect. 2.3 below). As we will show, they (together with the corresponding $r(p)$ with respect to reduced tuples) determine the value of $E\left(m_{1}, m_{2}, \ldots, m_{r}\right)$.

\section{Lemma 2.2.}

$$
E\left(p^{a_{1}}, p^{a_{2}}, \ldots, p^{a_{r}}\right)=(p-1)^{r-s+1} p^{v} h_{s}(p),
$$

where the multiplicity $s$ and the exponents $a_{j}$ are subject to (7), $v$ is determined by (8) and $h_{s}(x), s \geq 1$, is the following polynomial of $x$ of degree $s-2($ for $s>1)$ :

$$
h_{s}(x)=\frac{(x-1)^{s-1}+(-1)^{s}}{x} .
$$

Proof. We have

$$
E\left(p^{a_{1}}, \ldots, p^{a_{r}}\right)=\frac{1}{p^{a}} \sum_{k=1}^{p^{a}} \Phi\left(k, p^{a_{1}}\right) \cdots \Phi\left(k, p^{a_{r}}\right) .
$$

By (6), the first factor in these terms vanishes unless $k=d p^{a-1}$. So that we may restrict ourselves to such $k$ only, where $d=1,2, \ldots, p$. Again by (6), we get (for all $s$ including $s=1$ )

$$
E\left(p^{a_{1}}, \ldots, p^{a_{r}}\right)=\frac{1}{p^{a}}\left(\sum_{d=1}^{p-1} \Phi\left(d p^{a-1}, p^{a_{1}}\right) \cdots \Phi\left(d p^{a-1}, p^{a_{r}}\right)\right.
$$




$$
\begin{gathered}
\left.+\Phi\left(p^{a}, p^{a_{1}}\right) \cdots \Phi\left(p^{a}, p^{a_{r}}\right)\right) \\
=\frac{1}{p^{a}}\left((p-1) \Phi\left(p^{a-1}, p^{a_{1}}\right) \cdots \Phi\left(p^{a-1}, p^{a_{r}}\right)+\Phi\left(p^{a}, p^{a_{1}}\right) \cdots \Phi\left(p^{a}, p^{a_{r}}\right)\right) \\
=\frac{1}{p^{a}}\left((p-1)\left(-p^{a-1}\right)^{s}(p-1)^{r-s} p^{a_{s+1}-1} \cdots p^{a_{r}-1}+(p-1)^{r} p^{a_{1}-1} \cdots p^{a_{r}-1}\right) \\
=\frac{1}{p^{a}}\left((p-1)(-1)^{s} p^{(a-1) s} p^{\sum_{j=s+1}^{r}\left(a_{j}-1\right)}(p-1)^{r-s}+(p-1)^{r} p^{\sum_{j=1}^{r}\left(a_{j}-1\right)}\right) \\
=\frac{1}{p^{a}}\left((p-1)(-1)^{s} p^{\sum_{j=1}^{r}\left(a_{j}-1\right)}(p-1)^{r-s}+(p-1)^{r} p^{\sum_{j=1}^{r}\left(a_{j}-1\right)}\right) \\
=\frac{1}{p^{a}} p^{\sum_{j=1}^{r}\left(a_{j}-1\right)}(p-1)^{r-s+1}\left((-1)^{s}+(p-1)^{s-1}\right) \\
=p^{-1+\sum_{j=2}^{r}\left(a_{j}-1\right)}(p-1)^{r-s+1}\left((-1)^{s}+(p-1)^{s-1}\right) \\
=(p-1)^{r-s+1} p^{v}\left(\frac{(-1)^{s}+(p-1)^{s-1}}{p}\right) .
\end{gathered}
$$

In particular,

$$
\begin{aligned}
& h_{1}(x)=0, \\
& h_{2}(x)=1, \\
& h_{3}(x)=x-2, \\
& h_{4}(x)=x^{2}-3 x+3, \\
& h_{5}(x)=(x-2)\left(x^{2}-2 x+2\right) .
\end{aligned}
$$

Note that by (10), $(x-2) \mid h_{s}(x)$ for odd $s$ and $h_{s}(2)=1$ for even $s$. Clearly $h_{s}(p) \geq 0$ for any $s$ and $p \geq 2$. Moreover, $h_{s}(p)=0$ if and only if $s=1$ or $p=2$ and $s$ is odd.

Corollary 2.3. (1) $E\left(p^{a_{1}}, p^{a_{2}}, \ldots, p^{a_{r}}\right)$ is non-negative and integer.

(2) $E\left(p^{a_{1}}, p^{a_{2}}, \ldots, p^{a_{r}}\right)$ vanishes if and only if $s=1$ or $p=2$ and $s$ is odd.

(3) Let $p$ be odd. $p \nmid E\left(p^{a_{1}}, p^{a_{2}}, \ldots, p^{a_{r}}\right)$ if and only if $a=1$ and $r>1$; in this case, $s=r$.

(4) $E\left(p^{a_{1}}, p^{a_{2}}, \ldots, p^{a_{r}}\right)$ is odd if and only if $p=2, a=1$ and $s=r$ is even.

Proof. Claims (1) and (2) are immediate from formulae (9) and (10).

Claims (3). Suppose that $p$ does not divide $E\left(p^{a_{1}}, p^{a_{2}}, \ldots, p^{a_{r}}\right)$. Then $E\left(p^{a_{1}}, p^{a_{2}}, \ldots, p^{a_{r}}\right) \neq 0$ and by formula (9), $v=0$. It follows from (8) that $a_{2}=\cdots=a_{r}=1$. But $a_{1}=1$ as well and $r>1$, otherwise $s=1$ and then by Claim (2), $E\left(p^{a_{1}}, p^{a_{2}}, \ldots, p^{a_{r}}\right)=0$. Thus, $a=a_{1}=1$ and $s=r$.

On the contrary, if $a=1$ then $a_{1}=a_{2}=\cdots=a_{r}=1$ as well. Thus, $s=r$ and by (8), $v=0$. But in the right-hand side of formula (9), neither $p-1$ nor $h_{s}(p)$ is divisible by $p$ for $p>2$ and $s>1$. Hence if $r>1$ then $p \nmid E\left(p^{a_{1}}, p^{a_{2}}, \ldots, p^{a_{r}}\right)$.

Claims (4). $E\left(p^{a_{1}}, p^{a_{2}}, \ldots, p^{a_{r}}\right)$ is even for odd $p$ since always $r-s+1>0$ and, thus, the factor $(p-1)^{r-s+1}$ in the right-hand side of formula (9) is even. Now suppose $p=2$. We have the same situation as in the proof of Claim (3) with the only distinction that $2 \mid h_{s}(2)$ iff $s$ is odd. 
2.2. Multiplicativity. Now we turn to the general case. Take a tuple $\left(m_{1}, m_{2}, \ldots, m_{r}\right)$ and let $a_{j}(p) \geq 0$ denote the exponent with which the prime $p$ divides $m_{j}$. For a prime $p \mid m$, denote

$$
\left\{m_{1}, m_{2}, \ldots, m_{r}\right\}_{p}=\left(p^{a_{1}(p)}, p^{a_{2}(p)}, \ldots, p^{a_{r}(p)}\right) .
$$

Now denote

$$
E_{p}\left(m_{1}, m_{2}, \ldots, m_{r}\right):=E\left(\left\{m_{1}, m_{2}, \ldots, m_{r}\right\}_{p}\right)=E\left(p^{a_{1}(p)}, p^{a_{2}(p)}, \ldots, p^{a_{r}(p)}\right) .
$$

Then

$$
E_{p}\left(m_{1}, m_{2}, \ldots, m_{r}\right)=E\left(\left\langle p^{a_{1}(p)}, p^{a_{2}(p)}, \ldots, p^{a_{r}(p)}\right\rangle\right),
$$

where the angled brackets mean the removal of all the arguments equal to 1 , that is, the right-hand function contains a reduced set of arguments.

Proposition 2.4. $E(\emptyset)=E(1,1, \ldots, 1)=1$, and for $m>1$,

$$
E\left(m_{1}, m_{2}, \ldots, m_{r}\right)=\prod_{p \mid m \text { prime }} E_{p}\left(m_{1}, m_{2}, \ldots, m_{r}\right) .
$$

In other words, $E\left(m_{1}, m_{2}, \ldots, m_{r}\right)$ is a multiplicative function.

An arithmetic function $g=g\left(m_{1}, m_{2}, \ldots, m_{r}\right)$ of $r \geq 1$ arguments is called semi-multiplicative (see, e.g., [Hau88, Rea66]) if

$$
g\left(m_{1}, m_{2}, \ldots, m_{r}\right)=g\left(m_{1}^{\prime}, m_{2}^{\prime}, \ldots, m_{r}^{\prime}\right) \cdot g\left(m_{1}^{\prime \prime}, m_{2}^{\prime \prime}, \ldots, m_{r}^{\prime \prime}\right)
$$

whenever $m_{j}=m_{j}^{\prime} m_{j}^{\prime \prime}, j=1,2, \ldots, r$, and $\left(M^{\prime}, M^{\prime \prime}\right)=1$ where $M^{\prime}=$ $\prod_{j} m_{j}^{\prime}$ and $M^{\prime \prime}=\prod_{j} m_{j}^{\prime \prime}$. This function is called multiplicative if, moreover, $g(\underbrace{1,1, \ldots, 1}_{r})=1$. Two most known examples of symmetric multivariate multiplicative functions are $\operatorname{lcm}()$ and $\operatorname{gcd}()$.

$\Phi(k, n)$ is multiplicative as a function of $n$ and is periodic in $k$ modulo $n$ (it possesses, in fact, stronger properties but we do not need to use them here). Therefore Proposition 2.4 is straightforward from the following more general assertion.

Lemma 2.5. Let a bivariate arithmetic function $f(k, n)$ be semimultiplicative in the argument $n$ and periodic in $k$ modulo $n$. Given natural numbers $M, m_{1}, m_{2}, \ldots, m_{r}$, where $m_{j} \mid M$ for all $j$, the function

$$
F\left(m_{1}, m_{2}, \ldots, m_{r}\right)=\frac{1}{M} \sum_{k=1}^{M} f\left(k, m_{1}\right) f\left(k, m_{2}\right) \cdots f\left(k, m_{r}\right)
$$

is semi-multiplicative (with respect to all its arguments).

Proof. By the same reasons as in the Introduction, $F\left(m_{1}, m_{2}, \ldots, m_{r}\right)$ does not depend on $M$ (provided $m_{j} \mid M, j=1,2, \ldots, r$ ). So that, without loss of generality, we set $M=\prod_{j}^{r} m_{j}$. Suppose $m_{j}=m_{j}^{\prime} m_{j}^{\prime \prime}, j=1,2, \ldots, r$, where $\left(m_{i}^{\prime}, m_{j}^{\prime \prime}\right)=1$ for all $i, j$. Denoting $M^{\prime}=\prod_{j}^{r} m_{j}^{\prime}$ and $M^{\prime \prime}=\prod_{j}^{r} m_{j}^{\prime \prime}$ consider the product

$$
F\left(m_{1}^{\prime}, \ldots, m_{r}^{\prime}\right) \cdot F\left(m_{1}^{\prime \prime}, \ldots, m_{r}^{\prime \prime}\right)
$$




$$
=\frac{1}{M^{\prime}} \sum_{k^{\prime}=1}^{M^{\prime}} f\left(k^{\prime}, m_{1}^{\prime}\right) \cdots f\left(k^{\prime}, m_{r}^{\prime}\right) \cdot \frac{1}{M^{\prime \prime}} \sum_{k^{\prime \prime}=1}^{M^{\prime \prime}} f\left(k^{\prime \prime}, m_{1}^{\prime \prime}\right) \cdots f\left(k^{\prime \prime}, m_{r}^{\prime \prime}\right) .
$$

We claim that, regardless of the choice of all $m_{j}=m_{j}^{\prime} m_{j}^{\prime \prime}$, the equality $F\left(m_{1}, \ldots, m_{r}\right)=F\left(m_{1}^{\prime}, \ldots, m_{r}^{\prime}\right) \cdot F\left(m_{1}^{\prime \prime}, \ldots, m_{r}^{\prime \prime}\right)$ holds. Notice first that like $F\left(m_{1}, \ldots, m_{r}\right)$, the product $F\left(m_{1}^{\prime}, \ldots, m_{r}^{\prime}\right) \cdot F\left(m_{1}^{\prime \prime}, \ldots, m_{r}^{\prime \prime}\right)$ contains $M=M^{\prime} M^{\prime \prime}$ terms. Thus, all we need is to establish a bijection between the terms of the two functions and to show the equality of the corresponding terms.

Since the function $f(k, m)$ is periodic in $k$ modulo $m$, it is periodic modulo $M$, as well, if $m \mid M$, i.e., $f\left(k_{1}, m\right)=f\left(k_{2}, m\right)$ whenever $k_{1} \equiv k_{2}(\bmod M)$.

Consider a 'generic' term in $F\left(m_{1}^{\prime}, \ldots, m_{r}^{\prime}\right) \cdot F\left(m_{1}^{\prime \prime}, \ldots, m_{r}^{\prime \prime}\right)$ :

$$
t\left(k^{\prime}, k^{\prime \prime}\right):=f\left(k^{\prime}, m_{1}^{\prime}\right) \cdots f\left(k^{\prime}, m_{r}^{\prime}\right) \cdot f\left(k^{\prime \prime}, m_{1}^{\prime \prime}\right) \cdots f\left(k^{\prime \prime}, m_{r}^{\prime \prime}\right) .
$$

We look for $k$ such that $t\left(k^{\prime}, k^{\prime \prime}\right)=t(k, k)$. Take $k$ satisfying

$$
\begin{aligned}
& k \equiv k^{\prime}\left(\bmod M^{\prime}\right) \\
& k \equiv k^{\prime \prime}\left(\bmod M^{\prime \prime}\right) .
\end{aligned}
$$

Since $\left(M^{\prime}, M^{\prime \prime}\right)=1$, by the Chinese remainder theorem, such a $k$ does exist and is unique modulo $M$. Now, due to the periodic property, $f\left(k^{\prime}, m_{j}^{\prime}\right)=$ $f\left(k, m_{j}^{\prime}\right)$ and $f\left(k^{\prime \prime}, m_{j}^{\prime \prime}\right)=f\left(k, m_{j}^{\prime \prime}\right)$ for $j=1,2, \ldots, r$. Hence $t\left(k^{\prime}, k^{\prime \prime}\right)=$ $t(k, k)$ as required. Therefore by the semi-multiplicativity of $f(k, m)$ in $m$ we have

$t\left(k^{\prime}, k^{\prime \prime}\right)=f\left(k, m_{1}^{\prime}\right) \cdots f\left(k, m_{r}^{\prime}\right) \cdot f\left(k, m_{1}^{\prime \prime}\right) \cdots f\left(k, m_{r}^{\prime \prime}\right)=f\left(k, m_{1}\right) \cdots f\left(k, m_{r}\right)$.

It is clear that the established correspondence between $k$ and pairs $k^{\prime}, k^{\prime \prime}$ is a bijection between the sets $[1, M]$ and $\left[1, M^{\prime}\right] \times\left[1, M^{\prime \prime}\right]$, what gives rise to the required bijection between the terms of $F\left(m_{1}, m_{2}, \ldots, m_{r}\right)$ and those of $F\left(m_{1}^{\prime}, \ldots, m_{r}^{\prime}\right) \cdot F\left(m_{1}^{\prime \prime}, \ldots, m_{r}^{\prime \prime}\right)$.

Corollary 2.6. If $m_{1}=m_{1}^{\prime} m_{1}^{\prime \prime}$, where $\left(m_{1}^{\prime}, m_{1}^{\prime \prime}\right)=1$, then

$$
E\left(m_{1}, m_{2}, \ldots, m_{r}\right)=E\left(m_{1}^{\prime}, m_{1}^{\prime \prime}, m_{2}, \ldots, m_{r}\right) .
$$

Indeed, $E_{p}\left(m_{1}, m_{2}, \ldots, m_{r}\right)=E_{p}\left(\left\langle m_{1}^{\prime}, m_{1}^{\prime \prime}, m_{2}, \ldots, m_{r}\right\rangle\right)$ for all prime $p$.

Therefore, increasing $r$, one can split the arguments of $E\left(m_{1}, m_{2}, \ldots, m_{r}\right)$ into their primary factors:

$$
E\left(m_{1}, m_{2}, \ldots, m_{r}\right)=E\left(p^{a_{j}(p)}: p \mid m \text { prime, } j=1,2, \ldots, r, a_{j}(p) \geq 1\right) .
$$

Corollary 2.7. The values of $E\left(m_{1}, m_{2}, \ldots, m_{r}\right)$ are non-negative integers.

2.3. Main formulae. Given a tuple $\left(m_{1}, m_{2}, \ldots, m_{r}\right)$ with

$$
\operatorname{lcm}\left(m_{1}, m_{2}, \ldots, m_{r}\right)=m=\prod_{p \text { prime }} p^{a(p)},
$$

define for $p \mid m$, the parameters $s(p)$ and $v(p)$ that generalize the ones introduced in formulae (7) and (8):

$$
s(p):=\left|\left\{j: a_{j}(p)=a(p), j=1,2, \ldots, r\right\}\right|
$$


and

$$
v(p):=\sum_{\substack{j=1,2, \ldots, r \\ a_{j}(p) \geq 1}}\left(a_{j}(p)-1\right)-a+1 .
$$

Moreover, we count the arguments $m_{j}$ divisible by $p$, that is, the ones with $a_{j}(p)>0$ :

$$
r(p):=\left|\left\{m_{j}: p \mid m_{j}, j=1,2, \ldots, r\right\}\right| .
$$

The next theorem follows directly from (9) and (14) and gives rise to an explicit alternating-free formula for calculating $E\left(m_{1}, m_{2}, \ldots, m_{r}\right)$.

\section{Theorem 2.8.}

$$
E_{p}\left(m_{1}, m_{2}, \ldots, m_{r}\right)=(p-1)^{r(p)-s(p)+1} p^{v(p)} h_{s(p)}(p)
$$

and

$$
E\left(m_{1}, m_{2}, \ldots, m_{r}\right)=\prod_{p \mid m \text { prime }}(p-1)^{r(p)-s(p)+1} p^{v(p)} h_{s(p)}(p),
$$

where the parameters $s(p), v(p)$ and $r(p)$ are defined, respectively, by formulae (17), (18) and (19) and the polynomial $h_{s}(x)$ is defined by (10).

2.4. Further properties. According to (21), the value of the function $E\left(m_{1}, m_{2}, \ldots, m_{r}\right)$ is determined by the set of prime divisors $p \mid m$ and the parameters $s(p), v(p)$ and $r(p)$, where $r \geq r(p) \geq s(p) \geq 1$ and $v(p) \geq 0$. In particular, $E\left(m_{1}, m_{2}, \ldots, m_{r}\right)$ does not depend directly on $a(p)$ and $m$ (indirectly, however, $a(p)$ contributes into $v(p)$ ). Note also that $E\left(m_{1}, m_{2}, \ldots, m_{r}\right)$ does not depend on $r(2)$ as formulae (20) and (18) show; so that if $4 \mid m$, we may ignore the contributors $2^{1}$, that is, $a_{j}(2)=1$.

Corollary 2.9. (1) $\phi(m)$ divides $E\left(m_{1}, m_{2}, \ldots, m_{r}\right)$.

(2) $E\left(m_{1}, m_{2}, \ldots, m_{r}\right)=\phi(m)$ if and only if for every prime $p \mid m$, one of the following conditions holds:

$$
\left\langle\left\{m_{1}, m_{2}, \ldots, m_{r}\right\}_{p}\right\rangle=\left(p^{a(p)}, p^{a(p)}\right)
$$

or $p=3$ and

$$
\left\langle\left\{m_{1}, m_{2}, \ldots, m_{r}\right\}_{3}\right\rangle=(3,3,3)
$$

or $p=2$ and (up to reordering)

$$
\left\langle\left\{m_{1}, m_{2}, \ldots, m_{r}\right\}_{2}\right\rangle=(2^{a(2)}, 2^{a(2)}, \underbrace{2,2, \ldots, 2}_{r(2)-2}),
$$

where $a(2) \geq 1, r(2) \geq 3$ and $r(2)$ is even if $a(2)=1$.

Proof. Claim (1). Recall that $\phi\left(p^{a(p)}\right)=(p-1) p^{a(p)-1}$. Now, for any $p \mid m$, it follows directly from the definitions that $r(p)-s(p)+1 \geq 1$. Besides, $v(p) \geq a(p)-1$ if $E_{p}\left(m_{1}, m_{2}, \ldots, m_{r}\right) \neq 0$ since in this case, $s(p)>1$ by Corr. 2.3 (2). Thus, in (18), the term corresponding to $j=2$ is equal to $a(p)-1$. Therefore, by $(20), \phi\left(p^{a(p)}\right) \mid E_{p}\left(m_{1}, m_{2}, \ldots, m_{r}\right)$, and we are done by (14) and the multiplicativity of $\phi$. 
Claim (2). It is clear from (10) that $p$ does not divide $h_{s}(p)$ for $s>1$, nor does $p-1$ for $p>2$. Therefore by $(21), E\left(m_{1}, m_{2}, \ldots, m_{r}\right)=\phi(m)$ if and only if

$$
\begin{gathered}
r(p)-s(p)+1=1, \\
v(p)=a(p)-1
\end{gathered}
$$

and

$$
h_{s(p)}(p)=1
$$

for every prime $p \mid m$. Suppose $p \geq 3$. The first equality implies that $\left\langle\left\{m_{1}, m_{2}, \ldots, m_{r}\right\}_{p}\right\rangle=\left(p^{a(p)}, p^{a(p)}, \ldots, p^{a(p)}\right)$ whence $v(p)=(r(p)-$ 1) $(a(p)-1)$. Then the second equality implies that $r(p)=2$ or $a(p)=1$. If $r(p)=s(p)=2$ then we get $h_{s(p)}(x)=1$. If $r(p) \neq 2$ but $a(p)=1$, then $h_{s}(p)=1$ is possible only in one exceptional case: $h_{3}(3)=1$. Indeed, $h_{s}(x)=1$ can be represented as the equation $y^{s-1}-y-1+(-1)^{s}=0$, where $y=x-1$. It has only one integer solution greater $1: y=2$ and $s=3$. This solution corresponds to the tuple $(3,3,3)$ as claimed.

Finally, consider $p=2$ if $2 \mid m . \quad h_{s}(2)=1$ if and only if $s$ is even. Now $v(2)=a(2)-1$, what means that $\left\langle\left\{m_{1}, m_{2}, \ldots, m_{r}\right\}_{2}\right\rangle=$ $\left(2^{a(2)}, 2^{a(2)}, 2,2, \ldots, 2\right)$ with $a(2) \geq 1$. If $a(2)>1$, then $s(2)=2$ is even; if $a(2)=1$, then $s(2)=r(2)$ has to be even by Corr. 2.3 (4).

Let us consider in more detail the behavior of the function $E$ for $r \leq 3$ arguments.

Corollary 2.10. (1) $E(m) \neq 0$ if and only if $m=1$.

(2) $E\left(m_{1}, m_{2}\right) \neq 0$ if and only if $m_{1}=m_{2}$.

(3) $E\left(m_{1}, m_{2}, m_{3}\right) \neq 0$ if and only if for any prime $p \mid m$, the numbers $m_{1}, m_{2}$ and $m_{3}$ (in some order) are divided by $p$ with the exponents $a(p) \geq$ $b(p) \geq c(p)$ that meet one of the following conditions:

- $a(p)=b(p)>c(p)>0$;

- $a(p)=b(p)>c(p)=0$;

- $a(p)=b(p)=c(p)>0$ and $p>2$.

Proof. The first claim is obvious. As to the second one, if $m_{1} \neq m_{2}$ then there is a prime $p \mid m$ such that $s(p)=1$. Therefore the last factor in formula (21) vanishes.

In claim (3), if $a(p)>b(p) \geq c(p)$ for some $p \mid m$, then $s(p)=1$ and the last factor $h_{s}$ in formula (20) vanishes. It also vanishes if $2 \mid m, p=2$ and $s(2)=3$, that is, $a(2)=b(2)=c(2)$.

Now, $E_{p}$ is evaluated by formula (9), and we obtain for the last case of Corr. 2.10,

$$
E_{p}\left(m_{1}, m_{2}, m_{3}\right)=\left\{\begin{array}{ll}
(p-1)^{2} p^{a(p)+c(p)-2} & \text { if } a(p)=b(p)>c(p)>0 \\
(p-1) p^{a(p)-1} & \text { if } a(p)=b(p)>c(p)=0 \\
(p-1)(p-2) p^{2 a(p)-2} & \text { if } a(p)=b(p)=c(p)>0
\end{array}\right\} .
$$

Given $m=\prod_{p \mid m} p^{a(p)}$, Corr.2.10 (3) and Prop.2.4 make it possible to easily observe all triples $m_{1}, m_{2}, m_{3}$ for which $E\left(m_{1}, m_{2}, m_{3}\right) \neq 0$. Namely, for any prime $p \mid m$ we first form a triple $T_{p}=\left\{p^{a(p)}, p^{a(p)}, p^{c(p)}\right\}$ with an arbitrary integer $c(p), 0 \leq c(p) \leq a(p)$. Then we form three sets $\mathcal{M}_{1}, \mathcal{M}_{2}$ 
and $\mathcal{M}_{3}$ by distributing the elements of each $T_{p}$ arbitrarily by them (so that, there are three different ways if $c(p)<a(p)$, and only one way otherwise). Now we obtain the desired numbers $m_{j}$ as the products of the elements in the corresponding $\mathcal{M}_{j}: m_{j}:=\prod_{p \mid m} p^{b}, j=1,2,3$, where $p^{b} \in \mathcal{M}_{j}$. It follows that there are totally $\prod_{p \mid m}(3 a(p)+1)$ such ordered triples.

Corollary 2.11. $f_{r}(m):=E(\underbrace{m, m, \ldots, m}_{r})$ is the multiplicative function of $m$ determined by the formula

$$
f_{r}\left(p^{a}\right)=(p-1) p^{(r-1)(a-1)} h_{r}(p), \quad p \text { prime, } a>0 .
$$

According to (23), $f_{r}(m), m>1$, can be represented as follows:

$$
f_{r}(m)=m^{r-1} \prod_{p \mid m \text { prime }} \frac{(p-1) h_{r}(p)}{p^{r-1}} .
$$

It follows that $E(\underbrace{m, m, \ldots, m}_{r})=0$ if and only if $m$ is even and $r$ is odd or $r=1$ and $m>1$. Besides Nic53,

$$
E(m, m)=\phi(m)
$$

(in other words, $f_{2}(m)=\phi(m)$ ). This bivariate instance of $E$ is the only non-trivial particular specimen of the function defined by formula (1) that I managed to find in the number-theoretic literature.

Likewise, relying upon formula (21), we could investigate other general properties of the function $E\left(m_{1}, m_{2}, \ldots, m_{r}\right)$. One such property significant in topological applications, namely non-vanishing, will be considered below in Theorem 3.9 (in the particular case $m=p^{a}$ we have already encountered it in Corr. 2.3 (2) and later).

\section{Combinatorial and topological motivations and applications}

3.1. Linear congruences (restricted partitions). The following statement provides a simple combinatorial interpretation for the function $E$ :

Lemma 3.1. MeN06 Let $M$ be a natural number and $m_{1}, m_{2}, \ldots, m_{r}$ divisors of $M$. Denote $d_{1}=\frac{M}{m_{1}}, d_{2}=\frac{M}{m_{2}}, \ldots, d_{r}=\frac{M}{m_{r}}$. Then the number of solutions of the system of equations

$$
\left.\begin{array}{rl}
x_{1}+x_{2}+\cdots+x_{r} & \equiv 0(\bmod M) \\
\left(x_{1}, M\right) & =d_{1} \\
\cdots & \\
\left(x_{r}, M\right)= & d_{r}
\end{array}\right\}
$$

in integers modulo $M$ does not depend on $M$ and is equal to $E=$ $E\left(m_{1}, m_{2}, \ldots, m_{r}\right)$.

One could use Lemma 3.1 to give another proof of Proposition 2.4. 
3.2. Jordan's function. A more profound combinatorial interpretation for the function $E\left(m_{1}, m_{2}, \ldots, m_{r}\right)$ is presented below in Theorem 3.3 and combines $E$ with the classical Jordan function. Recall Apo76, Coh59, Sch99] that the Jordan function of order $k$ is defined by

$$
\phi_{k}(n)=\sum_{d \mid n} d^{k} \mu\left(\frac{n}{d}\right)
$$

or, equivalently, as a multiplicative function, by

$$
\phi_{k}(n)=n^{k} \prod_{p \mid n \text { prime }}\left(1-p^{-k}\right) .
$$

In particular, $\phi_{1}=\phi$ (Euler's totient). Moreover, $\phi_{0}(1)=1$ and $\phi_{0}(n)=0$ for $n>1$. It follows that

$$
\phi(n) \mid \phi_{k}(n)
$$

for $k \geq 1$.

As can be noticed in the literature on arithmetic functions, it is rather typical to research the Ramanujan sums and their generalizations jointly with the Jordan functions (cf., e.g., [Coh59, McC60]).

Remark 3.2. It is worth observing that the Jordan function participates (expressly or implicitly) in reductive enumeration formulae for conjugacy classes of subgroups of some finitely generated groups. The oldest formula of this kind was derived by the author [Lis71 and can be represented as follows:

$$
N_{F_{r}}(n)=\frac{1}{n} \sum_{d \mid n} \phi_{(r-1) d+1}\left(\frac{n}{d}\right) M_{F_{r}}(d),
$$

where $F_{r}$ is a free group of rank $r, M_{G}(n)$ denotes the number of subgroups of index $n$ in a group $G$ and $N_{G}(n)$ denotes the number of conjugacy classes of such subgroups. Subsequent similar formulae obtained by A. Mednykh in the 1980s and later for $n$-index subgroups of the fundamental groups of closed surfaces (see [Med06, Med08, KwMN08, and Lis98, Sect.2 and 5]) also contain $\phi_{k}(n / d)$ as a factor, where $k$ is a linear function of $d$. A considerable rôle of the Jordan function in this context has been realized only recently.

\subsection{Orbifolds and cyclic automorphism groups of Riemann sur-} faces. In order to describe more sophisticated applications of $E$ we need to remind some notions of algebraic topology. Generally for automorphisms of Riemann surfaces and orbifolds we refer to [Sco83, Sect. 2] and [BuEGG90, Ch.3]. By an orbifold we mean here the quotient space of a closed orientable surface with respect to an action of a finite group of orientation-preserving automorphisms. Denote by $\operatorname{Orb}\left(\mathcal{S}_{\gamma} / G\right)$ the set of orbifolds arising as the quotient spaces by the actions of the group $G$ on a Riemann surface $\mathcal{S}_{\gamma}$ of genus $\gamma$. Any orbifold $\Omega \in \operatorname{Orb}\left(\mathcal{S}_{\gamma} / G\right)$ is a closed surface $\mathcal{S}_{g}$ with a finite set of distinguished branch points; a surface is a particular case of an orbifold, with the empty set of branch points. $\Omega$ is characterized by its signature $\left(g ; m_{1}, m_{2}, \ldots, m_{r}\right), r \geq 0$, where $2 \leq m_{j} \leq \ell, j=1,2, \ldots, r$, are the orders 
of its branch points and $|G|=\ell$. We denote $\Omega=\Omega\left(g ; m_{1}, m_{2}, \ldots, m_{r}\right)$. Following Mednykh and Nedela [MeN06, by cyclic orbifolds we mean orbifolds corresponding to the cyclic groups $G=\mathbb{Z}_{\ell}$.

Given an orbifold $\Omega=\Omega\left(g ; m_{1}, m_{2}, \ldots, m_{r}\right)$, define the orbifold fundamental group $\pi_{1}(\Omega)$ to be the (Fuchsian) group generated by $2 g+r$ generators $x_{1}, y_{1}, x_{2}, y_{2}, \ldots, x_{g}, y_{g}$ and $z_{1}, z_{2}, \ldots, z_{r}$ and satisfying the relations

$$
\prod_{i=1}^{g}\left[x_{i}, y_{i}\right] \prod_{j=1}^{r} z_{j}=1 \quad \text { and } \quad z_{j}^{m_{j}}=1, \quad j=1, \ldots, r
$$

where $[x, y]=x y x^{-1} y^{-1}$.

Actions of a group $G$ on a surface naturally correspond to epimorphisms from the fundamental group of the corresponding orbifold onto $G$.

3.4. Epimorphisms $\pi_{1}(\Omega) \rightarrow \mathbb{Z}_{\ell}$. An epimorphism from $\pi_{1}(\Omega)$ onto a cyclic group of order $\ell$ is called order-preserving if it preserves the orders of the periodical generators $z_{j}, j=1, \ldots, r$. Equivalently, order-preserving epimorphisms have torsion-free kernels; in the literature, such epimorphisms are often called smooth, see, e.g., MaM98. We denote by $\operatorname{Epi}_{\mathrm{o}}\left(\pi_{1}(\Omega), \mathbb{Z}_{\ell}\right)$ the set of order-preserving epimorphisms $\pi_{1}(\Omega) \rightarrow \mathbb{Z}_{\ell}$. Lemma 3.1 makes it possible to find their number $\left|\operatorname{Epi}_{\mathrm{o}}\left(\pi_{1}(\Omega), \mathbb{Z}_{\ell}\right)\right|$ :

Theorem 3.3. MeN06] The number of order-preserving epimorphisms from the fundamental group $\pi_{1}(\Omega)$ of the cyclic orbifold $\Omega=$ $\Omega\left(g ; m_{1}, m_{2}, \ldots, m_{r}\right) \in \operatorname{Orb}\left(\mathcal{S}_{\gamma} / \mathbb{Z}_{\ell}\right)$ onto the cyclic group $\mathbb{Z}_{\ell}$ is expressed by the following formula:

$$
\left|\operatorname{Epi}_{\mathrm{o}}\left(\pi_{1}(\Omega), \mathbb{Z}_{\ell}\right)\right|=m^{2 g} \phi_{2 g}(\ell / m) \cdot E\left(m_{1}, m_{2}, \ldots, m_{r}\right),
$$

where $m=\operatorname{lcm}\left(m_{1}, m_{2}, \ldots, m_{r}\right)$ and $\phi_{2 g}(m)$ is the Jordan function of order $2 g$.

In particular, for $r=0$,

$$
\left|\operatorname{Epi}_{\mathrm{o}}\left(\pi_{1}(\Omega(g ; \emptyset)), \mathbb{Z}_{\ell}\right)\right|=\phi_{2 g}(\ell)
$$

and for $g=0$,

$$
\left|\operatorname{Epi}_{\mathrm{o}}\left(\pi_{1}\left(\Omega\left(0 ; m_{1}, m_{2}, \ldots, m_{r}\right)\right), \mathbb{Z}_{\ell}\right)\right|=E\left(m_{1}, m_{2}, \ldots, m_{r}\right)
$$

if, and only if, $m=\ell$.

Here and subsequently we follow the convention that an arithmetic function vanishes for non-integer arguments (besides, we pay the reader's attention to a minor change in designations with respect to $E\left(m_{1}, \ldots, m_{r}\right)$ : by some reasons, from now on, the letter $\ell$ is used instead of $M)$.

Due to formula (30), we call $E\left(m_{1}, m_{2}, \ldots, m_{r}\right)$ the orbicyclic (multivariate arithmetic) function! 1 .

According to Corollary 2.9 (1) and formula (30), $\left|\operatorname{Epi}_{\mathrm{o}}\left(\pi_{1}(\Omega), \mathbb{Z}_{\ell}\right)\right|$ is divisible by $\phi(\ell)$, what is obvious combinatorially since $\phi(\ell)$ is the number of primitive elements (units) in the group $\mathbb{Z}_{\ell}$. Moreover, by (30),

$$
\left|\operatorname{Epi}_{\mathrm{o}}\left(\pi_{1}(\Omega), \mathbb{Z}_{\ell}\right)\right|=\phi(\ell)
$$

\footnotetext{
${ }^{1}$ Curiously, a similar but different term (orbicycle index polynomial) has been introduced in BlD05, again in connection with orbit enumeration.
} 
if and only if $g=0, m=\ell$ and $m_{1}, m_{2}, \ldots, m_{r}$ satisfy the conditions of Corollary 2.9 (2). Of course in some cases, this can be established directly from (30). For example, if $g=0$ and $r=2$, then it is clear from (30) that $\Omega=\Omega\left(0, m_{1}, m_{2}\right)$ exist if and only if $m_{1}=m_{2}=m$, and in this case $\pi_{1}(\Omega)=\mathbb{Z}_{m}$. Now, order-preserving epimorphisms from $\mathbb{Z}_{m}$ onto $\mathbb{Z}_{\ell}$ exist if and only if $m=\ell$. Finally it is obvious that $\left|\operatorname{Epi}_{\mathrm{o}}\left(\mathbb{Z}_{m}, \mathbb{Z}_{m}\right)\right|=\phi(m)$.

3.5. Riemann-Hurwitz equation. The signature of any orbifold $\Omega\left(g ; m_{1}, m_{2}, \ldots, m_{r}\right) \in \operatorname{Orb}\left(\mathcal{S}_{\gamma} / G\right)$ satisfies the famous Riemann-Hurwitz equation:

$$
2-2 \gamma=\ell\left(2-2 g-\sum_{j=1}^{r}\left(1-\frac{1}{m_{j}}\right)\right),
$$

where $\ell=|G|$. It is clear that in $(\mathrm{RH})$,

$$
g \leq \gamma
$$

The following well-known inequalities can be easily deduced directly from the Riemann-Hurwitz equation (cf. [ZiVC80, 4.14.27]):

Proposition 3.4. (1) If $\Omega\left(g ; m_{1}, m_{2}, \ldots, m_{r}\right) \in \operatorname{Orb}\left(\mathcal{S}_{\gamma} / G\right),|G|=\ell$, then

$$
\ell \leq 4 \gamma+2 \text { for } g \geq 2 \text {. }
$$

Thus (see [Har66, Bro90]),

$$
\ell \leq 4 \gamma+2 \quad \text { for } \quad \gamma \geq 2 \quad \text { (Wiman, 1895). }
$$

(2) For all $\gamma \geq 0$,

$$
r \leq 2 \gamma+2 .
$$

Proof. Denoting $f=\sum_{j=1}^{r}\left(1-1 / m_{j}\right)$, (RH) can be rewritten as follows: $4 \gamma+2=2 \ell(2 g+f-2)+6$. If $2 \ell(2 g+f-2)+6<\ell$, then $\ell(4 g+2 f-5)+6<0$ and $4 g+2 f-5<0$, which is possible only for $g=0,1$ since $f \geq 0$.

Wiman's inequality (33) is immediate from (31) and (32).

Given $\gamma$, it is easy to see by (RH) that $r$ is maximal whenever $g=0$ and $m_{j}=m=\ell=2$ for all $j$, in which case $r=2 \gamma+2$.

Remark 3.5. (1) There is a subtler restriction on $\ell$ [Bro90]:

$$
\phi(\ell) \leq 2 \gamma \quad \text { for } \quad \gamma \geq 2
$$

For example, it excludes the order $\ell=9$ for $\gamma=2$, which satisfy (33). The bound (35) excludes also prime $\ell>2 \gamma+1$.

(2) Wiman's bound (33) is also valid for $\gamma=1$ provided that $r \geq 1$.

3.6. Harvey's conditions for automorphisms of Riemann surfaces.

Theorem 3.6. [Har66] There exists an orbifold $\Omega\left(g ; m_{1}, m_{2}, \ldots, m_{r}\right) \in$ $\operatorname{Orb}\left(\mathcal{S}_{\gamma} / \mathbb{Z}_{\ell}\right)$, where $\ell \geq 2$ and $\gamma \geq 2$, if and only if its parameters satisfy the Riemann-Hurwitz equation $(\mathrm{RH})$ and the following conditions:

(H1) $\operatorname{lcm}\left(m_{1}, m_{2}, \ldots, m_{j-1}, m_{j+1}, \ldots, m_{r}\right)=m$ for every $j=$ $1,2, \ldots, r$, where $m=\operatorname{lcm}\left(m_{1}, m_{2}, \ldots, m_{r}\right) \quad$ (the lcm-condition);

(H2) $m$ divides $\ell$, and $m=\ell$ if $g=0$;

(H3) $r \neq 1$, and $r \geq 3$ if $g=0$; 
$(\mathrm{H} 4)$ if $m$ is even, then the number of $m_{j}$ divisible by the maximal power of 2 dividing $m$ is even.

Remark 3.7. Theorem 3.6 is valid for $\gamma=0,1$ as well with the following condition that supplements (H3) MeN06:

(H3a) $r=2$ if $\gamma=0$, and $r \in\{0,3,4\}$ if $\gamma=1$.

3.7. Further applications of the function $E$. Note that $f_{r}(m)$ (formula (23)) gives rise to the number of solutions of the congruences (25) when $d_{1}=d_{2}=\ldots=d_{r}=1$. This is a particular case of the system considered and solved in NiV54 and later in Coh55 (see also Coh59]), where an arbitrary $n$ stands in place of 0 in the right hand side of the congruence. This was done in terms of the Ramanujan sums as well. A related enumeration problem was considered in Ugr93.

A simple enumerative proof can be obtained for another familiar result of Harvey that supplements the bound (33):

Corollary 3.8. [Har66] $\operatorname{Orb}\left(\mathcal{S}_{\gamma} / \mathbb{Z}_{4 \gamma+2}\right) \neq \emptyset$ for every $\gamma$. In other words, an orientable surface of genus $\gamma$ possesses an (orientation-preserving) automorphism of order $\ell=4 \gamma+2$.

Proof. Indeed, $\Omega=\Omega(0 ; 4 \gamma+2,2 \gamma+1,2) \in \operatorname{Orb}\left(\mathcal{S}_{\gamma} / \mathbb{Z}_{4 \gamma+2}\right)$ exists since the triple $m_{1}=4 \gamma+2, m_{2}=2 \gamma+1, m_{3}=2$ satisfies the Riemann-Hurwitz equation (RH) with $\ell=m=4 \gamma+2$ and $g=0$. Now by (30),

$\left|\operatorname{Epi}_{\mathrm{o}}\left(\pi_{1}(\Omega), \mathbb{Z}_{4 \gamma+2}\right)\right|=E(4 \gamma+2,2 \gamma+1,2)=E(4 \gamma+2,4 \gamma+2)=\phi(4 \gamma+2)>0$.

Note that $\Omega=\Omega\left(g ; m_{1}, m_{2}, \ldots, m_{r}\right) \in \operatorname{Orb}\left(\mathcal{S}_{\gamma} / \mathbb{Z}_{\ell}\right)$ exists if and only if $\left|\operatorname{Epi}_{\mathrm{o}}\left(\pi_{1}(\Omega), \mathbb{Z}_{\ell}\right)\right| \neq 0$ (see [Har66, MaM98]). Now we can derive an enumerative counterpart of Theorem 3.6 .

Theorem 3.9. There exists an orbifold $\Omega\left(g ; m_{1}, m_{2}, \ldots, m_{r}\right) \quad \in$ $\operatorname{Orb}\left(\mathcal{S}_{\gamma} / \mathbb{Z}_{\ell}\right)$, where $\ell \geq 2$ and $m_{j} \geq 2$ for all $j=1, \ldots, r$, if and only if its parameters satisfy the Riemann-Hurwitz equation $(\mathrm{RH})$ and none of the following conditions is valid:

(E1) $m \nmid \ell$;

(E2) $g=0$ and $\ell>m$;

(E3) $s(p)=1$ for some odd prime $p \mid m$;

(E4) $2 \mid m$ and $s(2)$ is odd.

Proof. According to $(30)$, the inequality $\left|\operatorname{Epi}_{\mathrm{o}}\left(\pi_{1}(\Omega), \mathbb{Z}_{\ell}\right)\right| \neq 0$ is equivalent to the condition

$$
m^{2 g} \phi_{2 g}(\ell / m) \cdot E\left(m_{1}, m_{2}, \ldots, m_{r}\right) \neq 0 .
$$

Now, $m^{2 g} \neq 0$ and $\phi_{2 g}(d)=0$ if and only if $m \nmid \ell$ or $g=0$ and $d>1$ (see (27)). It is clear from (21) and Corollary 2.3 (2), that $E_{p}\left(m_{1}, m_{2}, \ldots, m_{r}\right)=0$ if and only if one of conditions (E3) and (E4) of the theorem holds.

We can compare Theorem 3.9 with Harvey's Theorem 3.6. (E1) and (E2) are equivalent to (H2). It is obvious that (E3) is equivalent to (H1) and (E4) 
is equivalent to (H4). As to condition (H3), by Corollary 2.10 (1), $r \neq 1$. Now for $r=2$, by Corollary 2.10 (2), we would have $m_{1}=m_{2}=m$ but this contradicts (RH) for $g=0$ and $\gamma \neq 1$. Finally, $r=0$ (in which case $m=1$ ) is also impossible by $(\mathrm{RH})$ for $g=0$ and $\gamma \neq 0$.

Remark 3.10. Additional references. A related but different enumeration problem with respect to automorphisms groups (not necessarily cyclic) of orientable surfaces has been considered and solved by C. Maclachlan and A. Miller MaM98. A simple formula (with an implicit participation of the function $\left.\phi_{k}(n) / \phi(n)\right)$ that connects $\left|\operatorname{Epi}\left(\pi_{1}(\Omega), \mathbb{Z}_{\ell}\right)\right|$ with the number of homomorphisms $\left|\operatorname{Hom}\left(\pi_{1}(\Omega), \mathbb{Z}_{\ell}\right)\right|$ has been obtained by G. Jones Jon95. Different number-theoretic aspects of automorphisms of surfaces partially related to Harvey's theorem were investigated by W. Chrisman [Chr06]; cf. also the paper [Sie07] by M. Sierakowski. In general, the enumerative approach developed here may have certain useful interactions with combinatorics of automorphism groups of Riemann surfaces investigated in numerous recent and older publications (cf., e.g., CosI04, Gil76, Woo07]).

3.8. Map enumeration. A (topological) map is a proper cell embedding of a finite connected planar graph (generally with loops and multiple edges) in an orientable surface. A map is called rooted if an edge-end (a vertex-edge incidence pair), is distinguished in it as its root. Unlike maps without a distinguished root (called unrooted maps), rooted maps do not have non-trivial automorphisms. Unrooted maps are considered here up to orientation-preserving isomorphism; i.e., as maps on a surface with a distinguished orientation. For a general combinatorial and algebraic theory of maps see [CoM92, JoS78]. Most of numerous results obtained so far for counting maps are concerned with rooted planar maps, that is, rooted maps on the sphere. Typically maps are counted with respect to the number of edges.

Let $\left(g ; m_{1}, m_{2}, \ldots, m_{r}\right)$ be a signature of an orbifold and $b_{i} \geq 0, i \geq 2$, denote the number of branch points with $m_{j}=i$. That is, up to reordering, $\left(g ; m_{1}, m_{2}, \ldots, m_{r}\right)=(g ; \underbrace{2,2, \ldots, 2}_{b_{2}}, \underbrace{3, \ldots, 3}_{b_{3}}, \ldots)=\left[g ; 2^{b_{2}} 3^{b_{3}} \ldots \ell^{b_{\ell}}\right]$, where brackets indicate to the use of the parameters $b_{i}$ rather than $m_{j}$. In these terms, the following theorem is valid:

Theorem 3.11. [MeN06] The number of unrooted maps with $n$ edges on a closed orientable surface $\mathcal{S}_{\gamma}$ of genus $\gamma$ is

$\Theta_{\gamma}(n)=\frac{1}{2 n} \sum_{\ell \mid 2 n} \sum_{\Omega}\left|\operatorname{Epi}_{\mathrm{o}}\left(\pi_{1}(\Omega), \mathbb{Z}_{\ell}\right)\right| \sum_{s=0}^{b_{2}}\left(\begin{array}{c}2 n / \ell \\ s\end{array}\right)\left(\begin{array}{c}\frac{n}{\ell}-\frac{s}{2}+2-2 g \\ b_{2}-s, b_{3}, \ldots, b_{\ell}\end{array}\right) \mathcal{N}_{g}\left(\frac{n}{\ell}-\frac{s}{2}\right)$,

where $\ell$ runs over the divisors of $2 n, \Omega=\Omega\left[g ; 2^{b_{2}} \ldots \ell^{b_{\ell}}\right]$ runs over the orbifolds in $\operatorname{Orb}\left(\mathcal{S}_{\gamma} / \mathbb{Z}_{\ell}\right), \mathcal{N}_{g}(n)$ denotes the number of rooted maps with $n$ edges on an orientable surface of genus $g$ (with $\mathcal{N}_{g}(n):=0$ if $n$ is not integer) and $\left(\begin{array}{c}n \\ b_{2}, b_{3}, \ldots, b_{\ell}\end{array}\right)$ is the multinomial coefficient.

A similar formula holds for non-isomorphic hypermaps [MeN07, MeN10]. 
The orbicyclic function $E\left(m_{1}, m_{2}, \ldots, m_{r}\right)$ participates in (36) through formula (30). As for counting arbitrary rooted maps on orientable surfaces we refer to the papers [BeC91, ArG99] (for any genus $g$, there exists a closed formula, which is remarkably simple, sum-free, in the planar case and becomes more and more cumbersome as $g$ grows); see also WaL72.

Theorem 3.11 is a far-reaching generalization of the formula derived by the author for arbitrary unrooted planar maps [Lis81] (see also [Lis98]). Combinatorially, the proof of (36) follows the general reductive scheme elaborated for planar maps: relying upon Burnside's (orbit counting) lemma, the enumeration of unrooted maps (of a certain class) is reduced to the enumeration of their corresponding rooted quotient maps (i.e., orbifold maps) with respect to all possible orientation-preserving automorphisms of the underlying surface. In the case of arbitrary planar maps, quotient maps are merely almost arbitrary planar maps themselves, and we had nothing to do with the functions $\left|\mathrm{Epi}_{\mathrm{o}}\right|$ and $E\left(m_{1}, m_{2}, \ldots, m_{r}\right)$ (naturally, we made use of Euler's $\phi(m)$ in place of them). Burnside's lemma explains the rôle of cyclic groups in Th. 3.11.

Given $\gamma$, the above formulae make it possible to list all admissible orbifolds $\Omega=\Omega\left(g ; m_{1}, m_{2}, \ldots, m_{r}\right) \in \operatorname{Orb}\left(\mathcal{S}_{\gamma} / \mathbb{Z}_{\ell}\right)$. In view of $(33)$ and (34), for $\gamma \geq 2$, the set $\bigcup_{\ell \geq 1} \operatorname{Orb}\left(\mathcal{S}_{\gamma} / \mathbb{Z}_{\ell}\right)$ is finite. On the contrary, for $\gamma=0,1$, there are two infinite families of orbifolds, namely, $\operatorname{Orb}\left(\mathcal{S}_{0} / \mathbb{Z}_{\ell}\right)=\{\Omega(0 ; \ell, \ell)\}$ and $\operatorname{Orb}\left(\mathcal{S}_{1} / \mathbb{Z}_{\ell}\right)=\{\Omega(1 ; \emptyset)\}, \ell=1,2, \ldots ;$ (of course, in (36) they are restricted to the finite set of divisors of $2 n$ ). Moreover, there are four other orbifolds for $\gamma=1$ with $g=0$ and $\ell=2,3,4,6$. Denote

$$
A(\gamma):=\left|\bigcup_{\ell \geq 1} \operatorname{Orb}\left(\mathcal{S}_{\gamma} / \mathbb{Z}_{\ell}\right)\right|
$$

where $\operatorname{Orb}\left(\mathcal{S}_{\gamma} / \mathbb{Z}_{1}\right)=\{\Omega(\gamma ; \emptyset)\}$, and let $A_{g}(\gamma)$ denote the cardinality of the subset of all orbifolds $\Omega\left(g ; m_{1}, m_{2}, \ldots, m_{r}\right)$ of genus $g$ in this set. In particular [Bro90, MeN06, KuK90],

$$
A(2)=10, A(3)=17, A(4)=25, A_{0}(2)=8, A_{0}(3)=12, A_{0}(4)=18 .
$$

Apparently these functions could be investigated in general basing upon the restrictions considered above.

Concluding remarks. (1) No publication on multivariate arithmetic functions constructed similarly to (1) (see formula (15)) was known to me until recently when the preprint [Min09] appeared, where its author N. Minami considered (in quite a different context) the rational-valued function $\frac{1}{m} \sum_{k=1}^{m}\left(k, m_{1}\right)\left(k, m_{2}\right) \cdots\left(k, m_{r}\right)$ and derived a simple formula for its calculation.

(2) Addressing the polynomial $h_{s}(x)$ and formula (10) it would be interesting to find an explanation to the following observation (belongs to Alexander Mednykh, oral communication): $h_{s}(x)=\frac{\chi\left(\mathbf{C}_{s}, x\right)}{x(x-1)}$, where $\chi\left(\mathbf{C}_{s}, x\right)$ denotes the chromatic polynomial of a cycle of length $s$ (concerning the chromatic polynomials of graphs we refer the reader, e.g., to Chapter IX of the monograph [Tut84, in particular, to the formula for the chromatic polynomials of the cycles given in Theorem IX.24). 


\section{REFERENCES}

[Apo76] T. M. Apostol, Introduction to Analytical Number Theory, Springer, Berlin-New York, 1976, 338 pp. MR0434929 (55 \#7892)

[ArG99] D. Arquès and A. Giorgetti, Énumération des cartes pointées sur une surface orientable de genre quelconque en fonction des nombres de sommets et de faces, $J$. Combin. Theory Ser. B 77:1 (1999), 1-24 (in French). MR1710529 (2000k:05137)

[BeC91] E. A. Bender and E. R. Canfield, The number of rooted maps on an orientable surface, J. Combin. Theory Ser. B 53:2 (1991), 293-299. MR1129556 (92g:05100)

[BlD05] H. Blandin and R. Díaz, Pólya theory for orbiquotient sets, Preprint ArXiv: math.CO/0506630 (2005), 11 pp.

[Bro90] S. A. Broughton, Classifying finite group actions on surfaces of low genus, J. Pure Appl. Algebra 69:3 (1990), 233-270. MR1090743 (92b:57021)

[BuC99] E. Bujalance and M. Conder, On cyclic groups of automorphisms of Riemann surfaces, J. London Math. Soc. (2) 59:2 (1999), 537-584. MR1709666 (2000g:20098)

[BuEGG90] E. Bujalance, J. J. Etayo, J. M. Gamboa and G. Gromadzki, Automorphism Groups of Compact Bordered Klein Surfaces, Lecture Notes in Mathematics 1439. Springer, Berlin, 1990, 201 pp. MR1075411 (92a:14018)

[Chr06] M. W. Chrisman, The number theory of finite cyclic actions on surfaces. $\mathrm{PhD}$ thesis, University of Hawaii at Manoa, Dec. 2006, 200 pp. Available at: http://www.math.hawaii.edu/ ${ }^{\sim}$ micah/mwc_thesis.pdf

[Coh55] E. Cohen, An extension of Ramanujan's sum. II. Additive properties, Duke Math. J. 22:11 (1955), 543-550. MR0072163 (17,238g)

[Coh59] E. Cohen, Trigonometric sums in elementary number theory, Amer. Math. Monthly 66:2 (1959), 105-117. MR0098704 (20 \#5159)

[CoM92] R. Cori and A. Machì, Maps, hypermaps and their automorphisms: a survey, I, II, III, Exposition. Math. 10:5 (1992), 403-467. MR1190182 (94a:57006)

[CosI04] A.F. Costa and M. Izquierdo, Symmetries of real cyclic $p$-gonal Riemann surfaces, Pacific J. Math. 213:2 (2004), 231-243. MR2036917 (2004k:30098)

[Gil76] J. Gilman, On conjugacy classes in the Teichmüller modular group, Michigan Math. J. 23:1 (1976), 53-63. MR0430320 (55 \#3325)

[Har66] W. J. Harvey, Cyclic groups of automorphisms of a compact Riemann surface, Quart. J. Math. Oxford Ser. (2) 17:1 (1966), 86-97. MR0201629 (34 \#1511)

[Hau88] P. Haukkanen, Classical arithmetical identities involving a generalization of Ramanujan's sum, Ann. Acad. Sci. Fenn., Ser. A, I Math. Dissertationes No.68 (1988), 69 pp. MR0964709 (90e:11013)

[Jon95] G. A. Jones, Enumaration of homomorphisms and surface-coverings, Quart. J. Math. Oxford Ser. (2) 46:184 (1995), 485-507. MR1366619 (96m:20059)

[JoS78] G. A. Jones and D. Singerman, Theory of maps on orientable surfaces, Proc. London Math. Soc. (3) 37:2 (1978), 273-307. MR0505721 (58 \#21744)

[KuK90] I. Kuribayashi and A. Kuribayashi, Automorphism groups of compact Riemann surfaces of genera three and four, J. Pure Appl. Algebra 65:3 (1990), 277-292. MR1072285 (92a:30041)

[KwMN08] J.H. Kwak, A.D. Mednykh and R. Nedela, Enumeration of orientable coverings of a non-orientable manifold, Discrete Math. Theor. Comput. Sci. Proc. AJ (2008), 215-226 (electronic) (20th Intern. Conf. "Formal Power Series and Algebr. Combin." FPSAC'08, Valparaiso-Viña del Mar, Chile).

[Lis71] V. A. Liskovets, Towards the enumeration of subgroups of a free group, Dokl. Akad. Nauk BSSR 15:1 (1971), 6-9 (in Russian). MR0297848 (45 \#6900)

[Lis81] V. A. Liskovets, A census of non-isomorphic planar maps, Colloq. Math. Soc. Janos Bolyai 25, North-Holland, Amsterdam-New York (1981), 479-494 (Proc. Conf. "Algebraic Methods in Graph Theory", Szeged, 1978). MR0642058 (83a:05073)

[Lis98] V.Liskovets, Reductive enumeration under mutually orthogonal group actions, Acta Appl. Math. 52 (1998), 91-120. MR1649693 (2000b:05012)

[Lis05] V. Liskovets, The 'orbicyclic' arithmetic function, Workshop "Graph Embeddings and Maps on Surfaces 2005” GEMS 2005, Šafárik Univ., Košice, Slovakia, IM 
Preprint, Ser. A, No.6/2005, p.12. Available at

http://umv.science.upjs.sk/preprints/dokumenty/A6-2005.pdf

[MaM98] C. Maclachlan and A. Miller, Generating functions for finite group actions on surfaces, Math. Proc. Cambridge Philos. Soc. 124:1 (1998), 21-49. MR1620568 (99e:57026)

[McC60] P. J. McCarthy, Some properties of the extended Ramanujan sums, Arch. Math. (Basel) 11:4 (1960), 253-258. MR0112868 (22 \#3714)

[Med06] A.D. Mednykh, A new method for counting coverings over manifold with a finitely generated fundamental group, Dokl. Math. 74:1 (2006) 496-502 (transl. from Dokl. Akad. Nauk 409:2 (2006), 158-162, in Russian). MR2348885

[Med08] A. Mednykh, Counting conjugacy classes of subgroups in a finitely generated group, J. Algebra 320:6 (2008), 2209-2217. MR2437498 (2009m:20042)

[MeN06] A. D. Mednykh and R. Nedela, Enumeration of unrooted maps with given genus, J. Combin. Theory Ser. B 96:5 (2006), 706-729. MR2236507 (2007g:05088)

[MeN07] A. Mednykh and R. Nedela, Enumeration of unrooted hypermaps, Electron. Notes Discrete Math. 28 (2007) 207-214. MR2323967

[MeN10] A. Mednykh and R. Nedela, Enumeration of unrooted hypermaps of a given genus, Discrete Math. 310:3 (2010), 518-526.

[Min09] N. Minami, On the random variable $\mathbb{N} \ni l \mapsto \operatorname{gcd}\left(l, n_{1}\right) \operatorname{gcd}\left(l, n_{2}\right) \ldots \operatorname{gcd}\left(l, n_{k}\right) \in \mathbb{N}$, Preprint ArXiv: math.NT/0907.0918v1 (2009), 7 pp.

[Nic53] C. A. Nicol, On restricted partitions and a generalization of the Euler $\varphi$ number and the Moebius function, Proc. Nat. Acad. Sci. U.S. A. 39:9 (1953), 963-968. MR0056638 (15,105a)

[NiV54] C. A. Nicol and H.S. Vandiver, A von Sterneck arithmetical function and restricted partitions with respect to modulus, Proc. Nat. Acad. Sci. U.S.A. 40:9 (1954), 825-835. MR0063399 (16,115c)

[Rea66] D. Rearick, Semi-multiplicative functions, Duke Math. J. 33:1 (1966), 49-53. MR0184897 (32 \#2368)

[Sch99] J. Schulte, Über die Jordansche Verallgemeinerung der Eulerschen Funktion, Results Math. 36:3-4 (1999), 354-364. MR1726214 (2000j:11007)

[Sco83] P.Scott, The geometries of 3-manifolds, Bull. London Math. Soc. 15:5 (1983), 401-487. MR0705527 (84m:57009)

[Sie07] M. Sierakowski, Sets of periods for automorphisms of compact Riemann surfaces, J. Pure Appl. Algebra 208:2 (2007), 561-574. MR2277696 (2007k:30078)

[Siv89] R. Sivaramakrishnan, Classical Theory of Arithmetic Functions. Monographs and Textbooks in Pure and Applied Mathematics, 126. Marcel Dekker, New York, 1989, 386 pp. MR0980259 (90a:11001)

[Tut84] W.T. Tutte, Graph theory. Encyclopedia of Mathematics and Its Applications, 21. Menlo Park, California, Addison-Wesley, Cambridge University Press, 1984, 333 pp. MR0746795 (87c:05001)

[Ugr93] D. Ugrin-Šparac, On a class of enumeration problems in additive arithmetics, $J$. Number Theory 45:2 (1993), 117-128. MR1242712 (94h:11099)

[WaL72] T. R.S. Walsh and A. B. Lehman, Counting rooted maps by genus. I, II, J. Combin. Theory Ser. B 13:3 (1972), 192-218; 13:2 (1972), 122-141. MR0314686 (47 \#3237), MR0314687 (47 \#3238)

[Woo07] A. Wootton, The full automorphism group of a cyclic p-gonal surface, J. Algebra 312:1 (2007), 377-396. MR2320463 (2008c:14043)

[ZiVC80] H. Zieschang, E. Vogt and H.-D. Coldewey, Surfaces and Planar Discontinuous Groups. Lecture Notes in Mathematics 835, Springer, Berlin, 1980, 334 pp. MR0606743 (82h:57002)

V. A. Liskovets: Institute of Mathematics, National Academy of Sciences, 220072, Minsk, BELARUS

E-mail address: liskov@im.bas-net.by 